\title{
Psychosoziale Betreuung von kindlichen und jugendlichen Opferzeugen vor Gericht
}

\begin{abstract}
Zusammenfassung: Kinder und Jugendliche sind als Opfer von Straftaten besonders gefährdet und als eher schwache Mitglieder in der Gesellschaft besonders zu schützen. Durch die Gerichtsverhandlung kommt eine besondere Belastung auf sie zu. Unwissenheit und Ängste sind bei kindlichen und jugendlichen Zeugen häufig, und es besteht die Gefahr einer sekundären Traumatisierung der Opfer durch einen emotional negativen Kontakt mit der gerichtlichen Institution. Ein seit zwei Jahren am Düsseldorfer Landgericht und Amtsgericht etabliertes Zeugenbetreuungsprogramm soll einer solchen Gefahr vorbeugen. Im Rahmen eines begleitenden wissenschaftlichen Forschungsprojektes wurden neben 53 erwachsenen Opferzeugen auch 47 Kinder und Jugendliche als Zeugen mit standardisierten Selbstund Fremdbeurteilungsbogen untersucht. Die Ergebnisse belegen eine große Belastung der jüngeren Zeugen durch die Verhandlung. Sie profitieren aber auch besonders von der qualifizierten professionellen Betreuung. Ängste und Stress konnten erfolgreich reduziert werden. Mit einer Zeugenbetreuung vor Gericht kann ein wichtiger Beitrag zur Verhinderung einer sekundären Viktimisierung kindlicher und jugendlicher Zeugen geleistet werden.
\end{abstract}

Psychosocial Intervention to Help Child and Adolescent Witnesses in Court: The risk of crimes being committed against children and adolescents is particularly high. These individuals should receive effective protection. Confronted with judicial proceedings, they are exposed to enormous amounts of stress. Often they are overwhelmed by feelings of fear and insecurity. Secondary victimization is likely to follow as a result of the negative experiences they encounter with the judicial system. A victim assistance program has been set up in the regional and district court of Düsseldorf in order to protect against such trauma. Forty-seven child and adolescent victims and 53 adult victims, all turned witnesses, took part in the program. Standardized questionnaires for mood and affect were applied. Results show that the younger witnesses perceive the experience of the court proceedings as stressful compared to adults. However, they also benefit mostly from the victim support services. Fear and stress were successfully alleviated. Qualified victim support interventions may play an important role in protecting children and adolescents in the courtroom from „secondary victimization“.

PPmP Psychother Psychosom med Psychol 2001; 51: 160-165 (c) Georg Thieme Verlag Stuttgart · New York

ISSN 0937-2032
Ute Habel, Frank Schneider

Psychiatrische Klinik der Heinrich-Heine-Universität Düsseldorf
Key words: Child and adolescent victims - Court - Victim care program

\section{Einleitung}

Besonders Frauen und Kinder sind in der Bevölkerung gefährdet, Opfer von Straftaten zu werden, hauptsächlich als Opfer von Gewaltdelikten und bei Straftaten gegen die sexuelle Selbstbestimmung. Für Opfer stellt die Situation einer Zeugenaussage vor Gericht eine besondere Belastung dar [1,2], denn sie sind vor Gericht häufig auf sich alleine gestellt und mögen ihre Zeugenaussage oft eher als nicht sehr einfühlsam gestaltetes Verhör empfinden. Die Gefahr einer solchen vernachlässigenden Behandlung von Opfern vor Gericht liegt in einer sekundären Traumatisierung. Negative Umweltreaktionen bzw. Falschbehandlung der Opfer können zu weiteren psychischen Folgeschäden führen, die über die durch die Straftat primär verursachten hinausgehen. Busse et al. [3] haben festgestellt, dass nach Angaben der Mütter ca. 60\% der kindlichen Zeugen starke Ängste angesichts der auf sie zukommenden Belastung einer Gerichtsverhandlung hatten. 75\% der Mütter betrachteten die Verhandlung als hohe Belastung für ihr Kind.

Potenzielle Stressfaktoren, die mit einer Gerichtsverhandlung auf die kindlichen Opfer zukommen, sind beispielsweise Wartezeiten auf ungemütlichen Gerichtsfluren, Unwissen bezüglich der Rollen, Aufgaben [4] und auch Handlungsmöglichkeiten in der Verhandlung. Daraus und auch aus einer antizipierten Bedrohung durch den Täter resultiert Angst. Während der Vernehmung werden Belastung und Stress hervorgerufen durch die Begegnung mit dem Angeklagten, aber häufiger auch durch die Tatsache, vor vielen fremden Menschen aussagen zu müssen oder wiederholt befragt zu werden.

Zeugenbegleitprogramme vor Gerichtsverhandlungen existieren jedoch hierzulande hauptsächlich als Pilotprojekte. So gibt es spezielle Zeugenbetreuungsprogramme für kindliche Opferzeugen in Kiel [5], Rostock [6] oder Magdeburg [7].

Eingegangen: 30.11.1999

Angenommen: 12.4 .2000 
Ein Modellprojekt in Düsseldorf wurde am Land- und Amtsgericht eingerichtet, um vorrangig Opferzeugen zur Verfügung zu stehen [1,2]. Langfristiges Ziel dieses Projektes war es, mit den Ergebnissen ein „Düsseldorfer Modell“ zu etablieren, das über die Erstellung eines Leitfadens die Einrichtung und Institutionalisierung von Zeugenbetreuungsprogrammen an deutschen Gerichten erlaubt. Mit der Einrichtung der Zeugenbetreuung bei Gericht wurde daher eine wissenschaftliche Begleitforschung vom Justizministerium Nordrhein-Westfalen gefördert, die den Aufbau der Zeugenbetreuung dokumentiert und evaluiert und den Nachweis ihrer Notwendigkeit und Wirksamkeit erbringt.

\section{Methoden}

\section{Voraussetzungen}

Für die erfolgreiche Umsetzung eines solchen Zeugenbetreuungsprogramms bei Gericht sind zwei Voraussetzungen unerlässlich: die Einrichtung geeigneter, gut gelegener Räume im Gerichtsgebäude und die Einstellung kompetenter, im psychosozialen Bereich qualifizierter Fachkräfte. In unserem Fall konnten zwei Diplom-Sozialpädagoginnen eingestellt werden, die sich seit der Eröffnung des Zimmers für Zeuginnen und Zeugen im Dezember 1997 vorrangig um Opferzeugen kümmern. Bei der Auswahl der Bewerberinnen spielten neben einem Diplom in Pädagogik oder Psychologie Kriterien wie Vorerfahrungen in diesem oder einem ähnlichen Bereich (z. B. Gerichtshilfe) bzw. Erfahrung mit Gesprächsführungstechniken, Krisenintervention und Beratung eine wichtige Rolle. Ausführliche Anforderungen an das Curriculum von Zeugenbetreuerinnen sind im Laufe des Projektes erarbeitet worden [2]. Es stehen zwei Räume zur Verfügung, der eigentliche Betreuungsraum mit guter Lage zu den wichtigsten Sitzungssälen und ein Büroraum, der ebenfalls zur Betreuung genutzt werden kann.

\section{Stichproben}

Ein allen Ladungen beigefügtes Informationsblatt klärt alle Zeugen über die Möglichkeit der Zeugenbetreuung auf. Über die Anklageschriften und Ladungsverfügungen aus den Ge- schäftsstellen informieren sich die Betreuerinnen über laufende und geplante Verfahren und machen die dort geladenen Opferzeugen zusätzlich mit einem persönlichen Anschreiben auf das Zimmer für Zeuginnen und Zeugen aufmerksam.

Im Rahmen der wissenschaftlichen Begleitforschung wurden alle betreuten Zeugen mit einem Protokollbogen erfasst; zusätzlich wurden innerhalb der Projektlaufzeit Verfahrensstichproben (insgesamt 100 Opferzeugen) gezogen, bei denen die Befindlichkeit der Zeugen zu unterschiedlichen Zeitpunkten, die Akzeptanz des Programmes bei den Verfahrensbeteiligten sowie die Auswirkungen auf die Verhandlung mit standardisierten psychologischen Erhebungsmethoden erfasst wurden. Ab einem Stichtag (1.1.1998) wurden diese Zeugen schriftlich über das Forschungsvorhaben informiert. Alle Verfahren, deren Zeugen zur freiwilligen Teilnahme bereit waren, wurden konsekutiv einbezogen. Dies kann möglicherweise zu einer systematischen Stichprobenselektion insofern führen, als nur kooperationsbereite Zeugen untersucht werden. Eine Kontrollgruppe von 50 nicht betreuten Zeugen wurde am Landgericht Essen rekrutiert (zu den Ergebnissen vgl. [2]). Die Stichprobendaten zeigt Tab. 1.

\section{Untersuchungsinstrumente}

Für die gesonderte Befragung der Opferzeugen wurden selbst entwickelte sowie etablierte, standardisierte Selbst- und Fremdbeurteilungsbogen eingesetzt:

State-Trait-Anxiety Inventory (STAI) [8], erfasst die Angst als emotionalen Zustand (State-Angst) und als relativ überdauerndes Persönlichkeitsmerkmal (Trait-Angst). Befindlichkeitsskalierung anhand von Kategorien und Eigenschaftswörtern (BSKE) [9], dient der mehrdimensionalen Beschreibung des momentanen Befindens. Beschwerdenliste (BL, CIPS) [10], erfasst körperliche und Allgemeinbeschwerden. Hamilton Depression Scale (HAMD) [11], Fremdbeurteilungsskala zur quantifizierten Beurteilung der Depressivität anhand von 21 Symptomen aufgrund eines Interviews. Allgemeiner SchweregradScore (ASGS) [12], Beurteilungsscore für die Einschätzung der psychiatrischen Symptomatik. Er hat eine Streubreite von 0

Tab. 1 Stichprobenvergleich: demographische Daten und Merkmale der Opferzeugen in ihrer Situation als Opferzeugen vor Gericht (am Verhandlungstag, Angaben in \%). Für die Ausgangsstichprobe wurde nur ein Fragebogen verwendet, so dass die Datenmenge hier eingeschränkt ist und einige Angaben fehlen.

\begin{tabular}{|c|c|c|c|}
\hline & \multirow{2}{*}{$\begin{array}{l}\text { Ausgangsstichprobe } \\
\mathrm{n}=1218\end{array}$} & \multicolumn{2}{|c|}{ gesondert untersuchte Opferzeugen } \\
\hline & & $\begin{array}{l}\text { erwachsene Zeugen } \\
\mathrm{n}=53\end{array}$ & $\begin{array}{l}\text { kindliche und jugendliche Zeugen } \\
n=47\end{array}$ \\
\hline Alter in Jahren & - & $39,1 \pm 13,9$ & $16,0 \pm 2,4$ \\
\hline Geschlechterverteilung (\%) & 45 Männer/55 Frauen & 34 Männer/66 Frauen & 51 Jungen, 49 Mädchen \\
\hline Nationalität (\%) & - & 89 Deutsche & 89 Deutsche \\
\hline zum ersten Mál Zeuge (\%) & 54 & 57 & 66 \\
\hline Begleitung (\%) & 51 & 52 & 81 \\
\hline davon professionelle Begleitung (\%) & 23 & 4 & 4 \\
\hline Angst vor Repressalien (\%) & - & 56 & 53 \\
\hline Erfahrung von Repressalien (\%) & - & 14 & 30 \\
\hline erfolgte Aussage (\%) & - & 82 & 67 \\
\hline Arztbesuch (\%) & - & 42 & 43 \\
\hline Medikation (\%) & - & 13 & 4 \\
\hline subjektiver Behandlungswunsch (\%) & - & 16 & 15 \\
\hline Behandlungsindikation (\%) & - & 30 & 31 \\
\hline
\end{tabular}


bis 4 , wobei 0 keine psychiatrische Auffälligkeit bedeutet und 4 eine schwere psychiatrische Störung kennzeichnet.

Mit einem kurzen Fragebogen wurden nach der Zeugenaussage des betroffenen Zeugen die übrigen Verfahrensbeteiligten (Vorsitzender Richter, Staatsanwalt, Verteidiger und Nebenklagevertreter) zum möglichen Einfluss der Zeugenbetreuung auf das Aussageverhalten, den Ablauf des Verfahrens und die psychische Befindlichkeit des Zeugen befragt.

\section{Untersuchungsablauf}

Die Opferzeugen wurden vor der Inanspruchnahme der Betreuung in einer etwa 20-minütigen Befragung mit den erwähnten Fragebogen untersucht. Anschließend wurden sie im Zeugenbetreuungszimmer von einer der beiden Zeugenbetreuerinnen bis zum Verhandlungsbeginn und zum Teil darüber hinaus betreut. Nach der Aussage bzw. Verhandlung wurden sie erneut, auch bezüglich der Einschätzung der Zeugenbetreuung, interviewt. Die Zeugenbetreuerinnen füllten ebenfalls einen Fragebogen zur Betreuung des Zeugen aus. Nach drei Monaten wurden katamnestische Befragungen mit diesen Opferzeugen $(n=66)$ durchgeführt.

\section{Statistische Analyse}

Analysen von Mittelwertsunterschieden wurden mit Varianzanalysen und t-Tests auf Signifikanz geprüft, sofern es sich um Häufigkeitsvergleiche handelte mit $\chi^{2}$-Tests (teilweise McNemar $\chi^{2}$-Test). Als Signifikanzniveau wurde dabei ein $\alpha$ von $5 \%$ gewählt.

\section{Ergebnisse}

\section{Nutzung des Zimmers für Zeuginnen und Zeugen}

In der Erhebungszeit (Dezember 1997 bis Ende Juni 1999) wurden nach Auswertung der Protokollbogen 1218 Zeugen betreut, davon $12 \%$ jüngere Opferzeugen von unter 10 bis 20 Jahren, $56 \%$ Jungen und 44\% Mädchen ( $1 \%$ unter 10 Jahren, $4 \%$ zwischen 10 und 14 Jahren, 7\% zwischen 15 und 20 Jahren). Der Anteil betreuter Opferzeugen von allen betreuten Personen liegt insgesamt bei $46 \%$. Die Betreuungsinhalte bei jüngeren Zeugen und ihre Inanspruchnahme werden aus Tab. 2 ersichtlich.

\section{Spezifische Untersuchung von kindlichen und jugendlichen Opferzeugen}

Unter den 100 Opferzeugen, die gesondert von uns zu mehreren Zeitpunkten befragt wurden, waren $88 \%$ Opfer schwerwiegender Deliktformen (Tab.3). In dieser Gruppe ist ein hoher Anteil von $47 \%$ an kindlichen und jugendlichen Zeugen im Alter von 11 bis 20 Jahren enthalten. Der Anteil an Körperverletzungen liegt bei ihnen wesentlich höher (53\%) als bei erwachsenen Zeugen (32\%, vgl. Tab. 3). Obwohl $81 \%$ dieser kindlichen Zeugen in Begleitung kamen, meist mit den Eltern, nahmen sie das Angebot einer professionellen Zeugenbetreuung wahr. Tab. 1 enthält weitere Merkmale der Zeugen.

Betrachtet man den Behandlungsbedarf und die medizinische Versorgung der kindlichen und jugendlichen Opferzeugen $(n=47)$, so ist festzustellen, dass $43 \%$ einen Arzt infolge der
Tab. 2 Betreuungsinhalte jüngerer Zeugen bei der Zeugenbetreuung (ausgewertet anhand der Protokollbogen von betreuten Zeugen; Mehrfachnennungen waren möglich).

\begin{tabular}{lrr}
\hline Betreuungsinhalt & Anzahl & $\%$ \\
\hline persönliches Gespräch & 193 & 30 \\
allgemeine Information über das Gerichtsverfahren & 160 & 25 \\
Überbrückung der Wartezeit & 102 & 16 \\
Begleitung zur Verhandlung & 65 & 10 \\
vorbereitende Begehung des Verhandlungssaales & 63 & 9 \\
Information über und Vermittlung von Hilfsangeboten & 9 & 1 \\
sonstige & 57 & 9 \\
gesamt & 649 & 100 \\
\hline
\end{tabular}

Tab. 3 Prozentuale Verteilung auf die verschiedenen Verfahrensinhalte, zu denen die erwachsenen und jüngeren Opfer als Zeugen geladen waren.

\begin{tabular}{|c|c|c|}
\hline & $\begin{array}{l}\text { Verfahrens } \\
\text { erwachsene } \\
\text { Zeugen } \\
(n=53)\end{array}$ & $\begin{array}{l}\text { inhalte }(\%) \\
\text { kindliche und } \\
\text { jugendliche } \\
\text { Zeugen } \\
(n=47)\end{array}$ \\
\hline gegen die sexuelle Selbstbestimmung & 23 & 23 \\
\hline Raub & 19 & 15 \\
\hline BTM & 4 & 0 \\
\hline Körperverletzung & 32 & 53 \\
\hline gegen das Leben & 11 & 0 \\
\hline sonstige Vermögensdelikte & 6 & 2 \\
\hline sonstige & 6 & 6 \\
\hline
\end{tabular}

körperlichen und/oder psychischen Tatverletzungen aufsuchten; $11 \%$ waren bei einem Psychiater oder Psychologen und ein geringer Teil ( $2 \%$ ) bei einer Beratungsstelle vorstellig gewesen. Infolge unserer Befragung konnte eine behandlungsbedürftige psychische Symptomatik am Verhandlungstag (ASGS) bei knapp einem Drittel der Zeugen unter 20 Jahren festgestellt werden (31\%). Der ASGS liegt hier mit einem Mittel von 0,85 recht niedrig. Betrachtet man nur die jüngeren Zeugen mit Behandlungsindikation, so liegt der mittlere Schweregradscore wesentlich höher $(2,7)$ und deutet auf mäßige psychische Störungen, die eine ärztliche bzw. psychiatrische Behandlung erforderlich machen würden. Die depressive Symptomatik (HAMD) der jüngeren Zeugen ist nicht klinisch auffällig $(5,64 \pm 8,80)$, ebenso die mittlere Gesamtbeeinträchtigung durch körperliche und Allgemeinbeschwerden (BL, $13,81 \pm 12,27)$.

Etwa je ein Drittel der kindlichen und jugendlichen Zeugen hatte bereits im Vorfeld der Verhandlung (54\%) telefonischen (26\%) und/oder persönlichen (38\%) Kontakt zu den beiden Zeugenbetreuerinnen gesucht. Die meisten suchten im Rahmen der Zeugenbetreuung Informationen über den Verhandlungssaal ( $82 \%$ ) oder das Gerichtsverfahren ( $80 \%$ ) oder führten ein persönliches Gespräch mit den Zeugenbetreuerinnen (63\%). Die Zeugenbetreuung ist zeitintensiv, durchschnittlich betrug sie bei Erwachsenen und auch jüngeren Zeugen etwa zweieinhalb Stunden pro Zeuge.

Die Aussage vor dem Strafgericht ist nur für einen geringen Teil der Zeugen erleichternd (14\% der jüngeren, $12 \%$ der erwachse- 
nen Zeugen), die Mehrheit empfindet sie als Belastung (60\%). Für die jüngeren Zeugen stellt sie jedoch weniger häufig (54\%) eine Belastung dar als für Erwachsene (65\%). Diese waren auch geringgradig eher mit ihrer Aussage zufrieden (74\%) als erwachsene Zeugen (68\%).

\section{Wirkung der Zeugenbetreuung auf die Befindlichkeit der jüngeren Zeugen}

Vor der Verhandlung waren ihren Angaben zufolge $87 \%$ der Kinder und Jugendlichen und 85\% der Erwachsenen sehr nervös bzw. nervös (Abb.1).

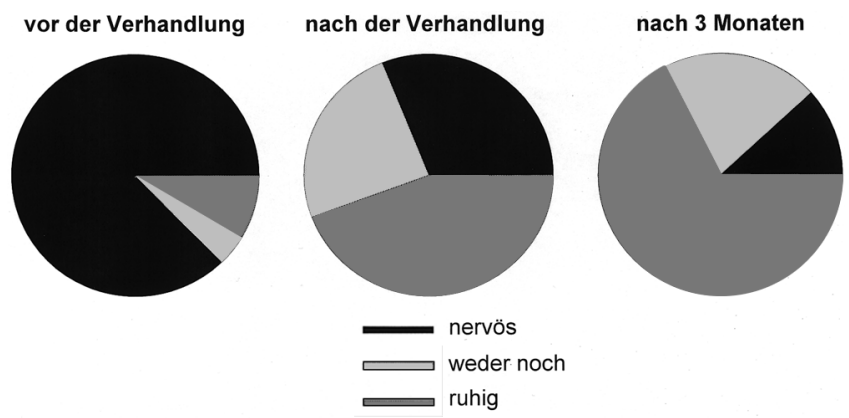

Abb.1 Subjektive Einschätzung der Aufregung und Nervosität zu verschiedenen Zeitpunkten bei kindlichen und jugendlichen Zeugen.

Diese Selbsteinschätzungen der Zeugen zeigen stärkere Belastung als es dem Eindruck entspricht, den die Zeugenbetreuerinnen hatten (69\% der jüngeren $\left(\chi^{2}=10,1, \mathrm{p}<0,05\right), 74 \%$ der erwachsenen Zeugen $\left(\chi^{2}=12,85, \mathrm{p}<0,05\right)$ waren nach Angaben der Betreuerinnen nervös). Im Anschluss an die Verhandlung gaben die kindlichen und jugendlichen Zeugen an, durch die Zeugenbetreuung ruhiger geworden $\mathrm{zu}$ sein (44\%). Dies trifft auch auf die Erwachsenen zu (43\%). Es war eine deutliche Abnahme der Angst (Abb. 2) bei jüngeren und erwachsenen Zeugen gleichermaßen (STAl, Zeitpunkt $\times$ Skala $\times$ Gruppe:

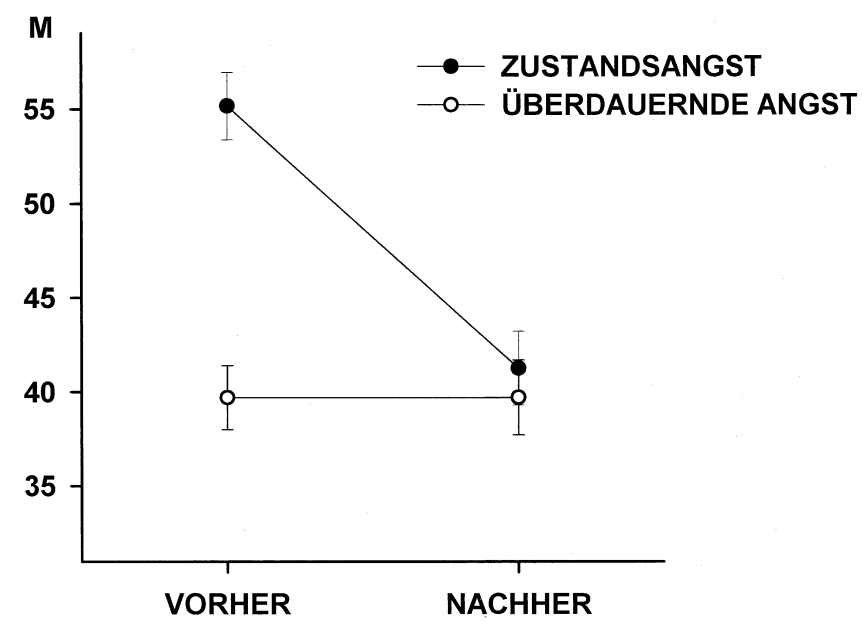

Abb. 2 Reduktion der aktuellen zustandsbedingten Angst nach der Betreuung und Verhandlung bei Kindern und Jugendlichen. Die generelle personenbedingte Ängstlichkeit ist dagegen bei beiden Erhebungszeitpunkten nicht verändert (STAI, $n=47$ ).
$\mathrm{F}(1,94)=0,04$, n. s.) messbar (STAI, Zeitpunkt $\times$ Skala: $F(1,94)=$ $65,10 ; p=0,0001)$.

Bei den kindlichen und jugendlichen Zeugen war anhand der BSKE eine Verbesserung des positiven Befindens $(\mathrm{t}(45)=$ $-3,48, p=0,001)$, des positiven Gefühls $(t(45)=-3,51$, $\mathrm{p}=0,001)$ sowie eine signifikante Zunahme der Zufriedenheit $(t(45)=-3,29, p=0,002)$, der Ausgeglichenheit $(t(45)=-3,20$, $\mathrm{p}=0,003)$, des Wohlgefühls $(\mathrm{t}(45)=-2,70, \mathrm{p}=0,01)$, des körperlichen Wohlbefindens $(t(45)=-2,19, p=0,03)$ und der Gutgestimmtheit $(t(45)=-4,43, p=0,0001)$ sowie eine Abnahme von negativem Befinden ( $t(45)=2,95, p=0,005)$, negativen $\mathrm{Ge}$ fühlen $(t(45)=3,09, \quad p=0,003)$, Erregung $(t(45)=5,19$, $\mathrm{p}=0,0001)$ und Angst $(\mathrm{t}(45)=4,77, \mathrm{p}=0,0001)$ feststellbar.

\section{Befragung der übrigen Prozessbeteiligten}

Die Befragung der anderen Prozessbeteiligten ergab, dass entgegen anfänglicher Vorbehalte dem Projekt gegenüber 93\% der Juristen von der Notwendigkeit einer solchen Betreuung bei Gericht überzeugt waren. Wenn auch viele angaben, dies nicht beurteilen zu können, so nahmen doch $42 \%$ der Verfahrensbeteiligten einen positiven Einfluss auf die Befindlichkeit der jüngeren Zeugen durch die Zeugenbetreuung wahr. Ursprüngliche Befürchtungen, die Zeugenbetreuung übe einen negativen Einfluss auf die Zeugenaussage aus, konnten anhand der Angaben der Verfahrensbeteiligten bei den jüngeren Zeugen nicht bestätigt werden, da durchschnittlich 59\% angaben, dies nicht beurteilen zu können oder äußerten, die Zeugenbetreuung habe keinen Einfluss auf das Aussageverhalten (7\% der Richter, 24\% der Staatsanwälte, 15\% der Verteidiger, 100\% der Nebenklagevertreter). Ähnliches galt bezüglich des Einflusses auf den Verfahrensablauf: Von nur einem Verteidiger wurde ein negativer Einfluss beschrieben, stattdessen nahmen immerhin $40 \%$ der übrigen Verfahrensbeteiligten einen positiven Einfluss wahr.

\section{Diskussion}

Die Ergebnisse belegen die rasche und positive Annahme der Zeugenbetreuung am Landgericht und Amtsgericht Düsseldorf. In einigen Bundesländern bestehen bereits speziell für kindliche Opferzeugen eingerichtete Zeugenbetreuungsprojekte: Die Bestandteile des Kieler Modellprojektes [5] reichen von der Kontaktaufnahme bis hin zur Nachbereitung der Hauptverhandlung. Infolge der Betreuung konnte eine Belastungsreduktion und eine Steigerung der Selbstsicherheit der Kinder in der Hauptverhandlung beobachtet werden. In Rostock [6] setzt die Betreuung bereits bei der Anzeigenerstattung ein und bietet die Möglichkeit zu vorbereitenden Gesprächen bis zur Verhandlung. Ähnlich breit gespannt ist auch das Magdeburger Interventionsprojekt [7].

Unsere Daten zeigen, dass angesichts der großen Nachfrage nach einer Betreuung bei männlichen Zeugen eine generelle Einschränkung auf Frauen oder Kinder nicht gerechtfertigt erscheint. Ein Anteil von $42 \%$ betreuten anderen Zeugen (darunter $23 \%$ kindliche und jugendliche Zeugen) belegt ferner das hohe Bedürfnis nach kompetenter Beratung und Unterstützung nicht nur bei Opferzeugen. Allerdings wurden „Berufszeugen“ wie Polizeibeamte oder Fahrkartenkontrolleure von einer Betreuung ausgeschlossen. 
Infolge der Zeugenbetreuung konnte eine deutliche Belastungsreduktion und Befindlichkeitsverbesserung bei den jüngeren Zeugen festgestellt werden. Ein Vergleich aller 100 gesondert untersuchten Opferzeugen mit nicht betreuten Zeugen eines Essener Landgerichtes $(n=50)$ zeigte, dass dieser Effekt bei betreuten Zeugen nicht alleine darauf zurückzuführen war, dass die Zeugen erleichtert darüber waren, dass Verhandlung und Aussage beendet sind. Die spezifische Wirksamkeit der Betreuung wurde an der sehr viel stärkeren und ausgeprägteren Befindlichkeitsveränderung vom Zustand vor der Verhandlung zu dem nach der Aussage bei betreuten Zeugen sichtbar [2].

Fischer [13] geht von andauernden Belastungssyndromen bei ca. 20-30\% der Opfer von Gewaltverbrechen aus. Unsere Ergebnisse zur Behandlungsindikation bei Kindern und Jugendlichen wie auch Erwachsenen bestätigen diese Schätzungen. $\mathrm{Zu}$ diesen dauerhaften Symptomen kommt die aktuelle Belastung durch die bevorstehende Gerichtsverhandlung hinzu. Die vor der Verhandlung geäußerte Nervosität scheint jedoch nicht in gleichem Maße ausgedrückt zu werden. Die Betreuerinnen nehmen weniger Nervosität besonders bei den jüngeren Zeugen war, als sie selbst angeben.

Bei Kindern wird häufig die wiederholte Befragung durch verschiedene fremde Personen als Belastungsfaktor im Zusammenhang mit der Ermittlung und Verhandlung genannt. Der Verzicht auf die Aussage wird daher als zeugenschonende Maßnahme angesehen. Bei nur einem Drittel der hier befragten kindlichen und jugendlichen Zeugen war dies der Fall. Die Aussage stellt für die überwiegende Mehrheit der Zeugen eine große Belastung dar (60\%), wenngleich dies von Kindern und Jugendlichen selbst weniger häufig berichtet wurde als von Erwachsenen. Auch die Zufriedenheit mit der Aussage war bei Zeugen unter 20 Jahren etwas häufiger zu finden als bei erwachsenen Zeugen. In einer Untersuchung von Busse et al. [3] gaben $80 \%$ der kindlichen Opferzeugen ohne Zeugenbetreuung an, die Aussage als belastend empfunden zu haben. Man kann vermuten, dass jüngere Zeugen besonders von der Zeugenbetreuung profitieren, indem infolge der Betreuung das aktuelle Belastungserleben durch die Aussage gesenkt werden kann und gleichzeitig die Zufriedenheit mit der Aussage gesteigert werden kann.

Dennoch sind unserem Eindruck nach Zeugen oft verärgert und enttäuscht, wenn auf ihre Aussage in der Verhandlung kurzfristig und besonders ohne Erklärung oder Angabe von Gründen verzichtet wird. $1 \mathrm{~m}$ Hinblick auf die Tatverarbeitung und auch aus psychotherapeutischen Gründen sollte daher bei geladenen Zeugen, die sich bereits im Gerichtsgebäude befinden, nicht generell versucht werden, dem Opfer die Aussage zu „ersparen“. Doch die bisherige Ergebnislage zur Wirkung der Zeugenaussage ist uneinheitlich. Einerseits wurden keine Unterschiede zwischen Kindern, die aussagen mussten und solchen, die nicht aussagten, berichtet [14], andererseits waren sieben Monate nach der Aussage mehr Verhaltensstörungen bei Kindern (im Vergleich zu solchen, die nicht aussagen mussten) zu finden, bei denen es in der Gerichtsverhandlung zu einer Aussage gekommen war [15]. Da es sich für einen großen Teil der Zeugen um die erste Aussage bei Gericht handelte, kann die Situation angsteinflößend, unbekannt und unkontrollierbar erscheinen. Der Kontrollverlust im Zusammenhang mit der Tat und den Ermittlungen kann charakteristische Reak- tionen des Aufgebens, der Hilflosigkeit und Depression zur Folge haben $[16,17]$. Die Zeugenbetreuung ist hier von entscheidender Relevanz, da sie im Vorfeld die nötigen Informationen, angemessene kognitive Bewältigungsstrategien und Kontrollmöglichkeiten liefern und soziale Unterstützung bieten kann. Dadurch werden Unsicherheiten verringert und Reaktionen der Hilflosigkeit vermieden.

Wie King et al. [18] feststellten, muss die effektive Möglichkeit der Stressreduktion nicht notwendigerweise in einer Vermeidung der Zeugenaussage liegen. Im Falle einer entsprechenden Vorbereitung und Unterstützung können die Kinder und Jugendlichen besser mit der Belastung umgehen. Die gute psychische Verfassung unserer Zeugen im Anschluss an die Verhandlung unterstreicht dies. Die positive Befindlichkeitsänderung der Zeugen infolge der Zeugenbetreuung kann als erster Erfolgsindikator bei der Verhinderung verfahrensinduzierter langfristiger Schädigungen im Sinne einer sekundären Traumatisierung dienen, auch wenn hier langfristige Effekte noch weitergehend mit Hilfe von Langzeitstudien untersucht werden müssen. Sie unterstreicht die Notwendigkeit der Einrichtung weiterer Zeugenbetreuungsstellen an Land- und Amtsgerichten.

\section{Danksagung}

Die Durchführung dieser Studie erfolgte aus Mitteln des Justizministeriums Nordrhein-Westfalen an F. S. (3003E-IIC.52/96). Wir danken Iris Beck, Petra Hammer, Siglinde Hinzberger, Gustav Marten, Stefanie Maurer, Heidrun Riedel, Mike Schmidt und Karin Schulze für ihre Mitarbeit und Unterstützung.

\section{Literatur}

${ }^{1}$ Schneider F, Weiss U. Psychosoziale Betreuung von Opferzeugen in Strafprozessen: Der Beginn der Düsseldorfer Zeugenbetreuungsstelle. Betrifft Justiz 1998; 55: $306-312$

2 Schneider F, Habel U. Psychosoziale Betreuung von Opferzeugen in Strafprozessen. Baden-Baden: Nomos, 2000

${ }^{3}$ Busse D, Volbert R, Steller M. Belastungserleben von Kindern in Hauptverhandlungen. Bonn: Bundesministerium für Justiz, 1996

${ }^{4}$ Wolf P. Was wissen Kinder und Jugendliche über Gerichtsverhandlungen? Regensburg: Roderer, 1997

${ }^{5}$ Köhnken G, Dannenberg U. Das schleswig-holsteinische Zeugenbegleitprogramm für Kinder. Praxis der Rechtspsychologie 1997; 7: $204-212$

${ }^{6}$ SELMA. Konzeption Mädchenprojekt „SELMA“-Rostock „Betreuung kindlicher ZeugInnen“. Trägerin: „Frauen helfen Frauen“. Rostock: (unveröffentlicht)

${ }^{7}$ Kavemann B. Abschlussbericht der wissenschaftlichen Begleitung des Magdeburger Interventionsprojektes für die Opfer sexueller Gewalt. Magdeburg: Leitstelle für Frauenpolitik des Landes Sachsen-Anhalt, 1996

${ }^{8}$ Laux L, Glanzmann P, Schaffner P, Spielberger CD. Fragebogen zur Erfassung von Angst und Ängstlichkeit. In: Spiegelberger CD, Gorsuch RL, Lushene RE: Deutsche Bearbeitung des State-TraitAnxiety-Inventory (STAI). Weinheim: Beltz, 1977

${ }^{9}$ Janke W, Debus G, Erdmann G, Hüppe M, Holler M. Befindlichkeitsskalierung anhand von Kategorien und Eigenschaftswörtern. BSKE (41). Würzburg: Psychologisches Institut (unveröffentlicht), 1998

${ }^{10}$ CIPS. Collegium Internationale Psychiatricae Scalarum. Internationale Skalen für Psychiatrie (4. Aufl.). Göttingen: Beltz, 1996

${ }^{11}$ Hamilton M. A rating scale for depression. J Neurol Neurosurg Psychiatry 1960; 23: 56-62 
${ }^{12}$ Cooper B. Probleme der Falldefinition und der Fallfindung. Nervenarzt 1978; 49: 437-444

${ }^{13}$ Fischer G, Becker-Fischer M, Düchting C. Neue Wege in der Hilfe für Gewaltopfer. Ergebnisse und Verfahrensvorschläge aus dem Kölner Opferhilfe Modell. Düsseldorf: Ministerium für Arbeit, Gesundheit und Soziales des Landes Nordrhein-Westfalen, 1998

${ }^{14}$ Oates RK, Lynch DL, Stern AE, O’Toole BI, Cooney G. The criminal justice system and the sexually abused child. Help or hindrance? Med J Aust 1995; 162: 126-130

15 Goodman GS, Taub EP, Jones DO, England P, Port LK, Rudy L, Prado L. Testifying in criminal court: emotional effects on child sexual assault victims. Monogr Soc Res Child Dev 1992; 57: 1 142

${ }^{16}$ Seligman MEP. Erlernte Hilflosigkeit (5. korr. Aufl.). Weinheim: Beltz, 1995

${ }^{17}$ Lazarus RS, Folkman S. Stress, appraisal and coping. New York: Springer, 1984

${ }^{18}$ King NM, Hunter WM, Runyan DK. Going to the court: the experience of child victims of intrafamilial sexual abuse. J Health Polit Policy Law 1988; 13: $705-721$

\section{Dr. Ute Habel}

Psychiatrische Klinik der Heinrich-Heine-Universität Düsseldorf Bergische Landstraße 2 40629 Düsseldorf

E-mail: habel@uni-duesseldorf.de 